\title{
The unusual emission line spectrum of I Zw 1
}

\author{
Monique Joly ${ }^{1}$, M.-P. Véron-Cetty ${ }^{2}$ \\ and P. Véron ${ }^{2}$ \\ ${ }^{1}$ Observatoire de Paris-Meudon, 5 place J. Janssen, F-92195 Meudon, France \\ email: monique.joly@obspm.fr \\ ${ }^{2}$ Observatoire de Haute Provence, CNRS, F-04870 Saint-Michel l'Observatoire, France \\ email: mira.veron@oamp.fr philippe.veron@oamp.fr
}

Abstract. A detailed analysis of the spectrum of I Zw 1 shows that the Narrow Line Region, unlike that of most Seyfert 1 galaxies, is a very low excitation region dominated by both permitted and forbidden Fe II lines. The physical conditions in this region are discussed.

\section{Introduction}

Our first aim in revisiting the optical spectrum of I Zw 1 was to build an accurate template of the Fe II emission, in the hope of improving that of Boroson \& Green (1992).

Indeed, most Seyfert 1s show strong Fe II line emission in their spectrum overlapping the other components. To unveil these components, the FeII contribution should be removed. In order to achieve this, an accurate Fe II template is necessary.

I Zw 1 is a bright NLS1 galaxy with strong Fe II emission. Consequently, it is a good candidate to extract a pure Fe II spectrum.

\section{Data Analysis}

We used very high $\mathrm{S} / \mathrm{N}$ medium resolution optical spectra of I Zw 1, obtained from the AAT and WHT data archives.

To identify all emission lines, the spectra were fitted by using a software originally written by E. Zuiderwijk, and described in Véron et al. (1980). The code has been substantially improved since, by M.-P. Véron-Cetty and P. Véron.

In this code, several redshift systems are allowed; their number is determined by trial and error. A Lorentz profile was assumed for the lines of the BLR, and a Gaussian profile for all other systems. Within each system, all lines have the same redshift, width and profile. Furthermore, the line intensity ratios of some forbidden lines were set to their theoretical values.

Through a least-square fitting, the code provides the values of the free parameters: the redshift and line width of each system, and the intensity of each line.

\section{The Emission Regions in the Nucleus of I $\mathrm{Zw}_{\mathrm{w}} 1$}

In agreement with previous studies, four redshift systems were needed in order to obtain a good fit to the observations.

- The Broad Line Region (FWHM $\sim 1100 \mathrm{~km} \mathrm{~s}^{-1}$ ) which emits the Fe II permitted lines from which we build the Fe II template. As commonly observed in Seyfert 1 galaxies, it emits also HI, He I, Na I D, Ti II and Si II lines. This region is slightly blueshifted by 


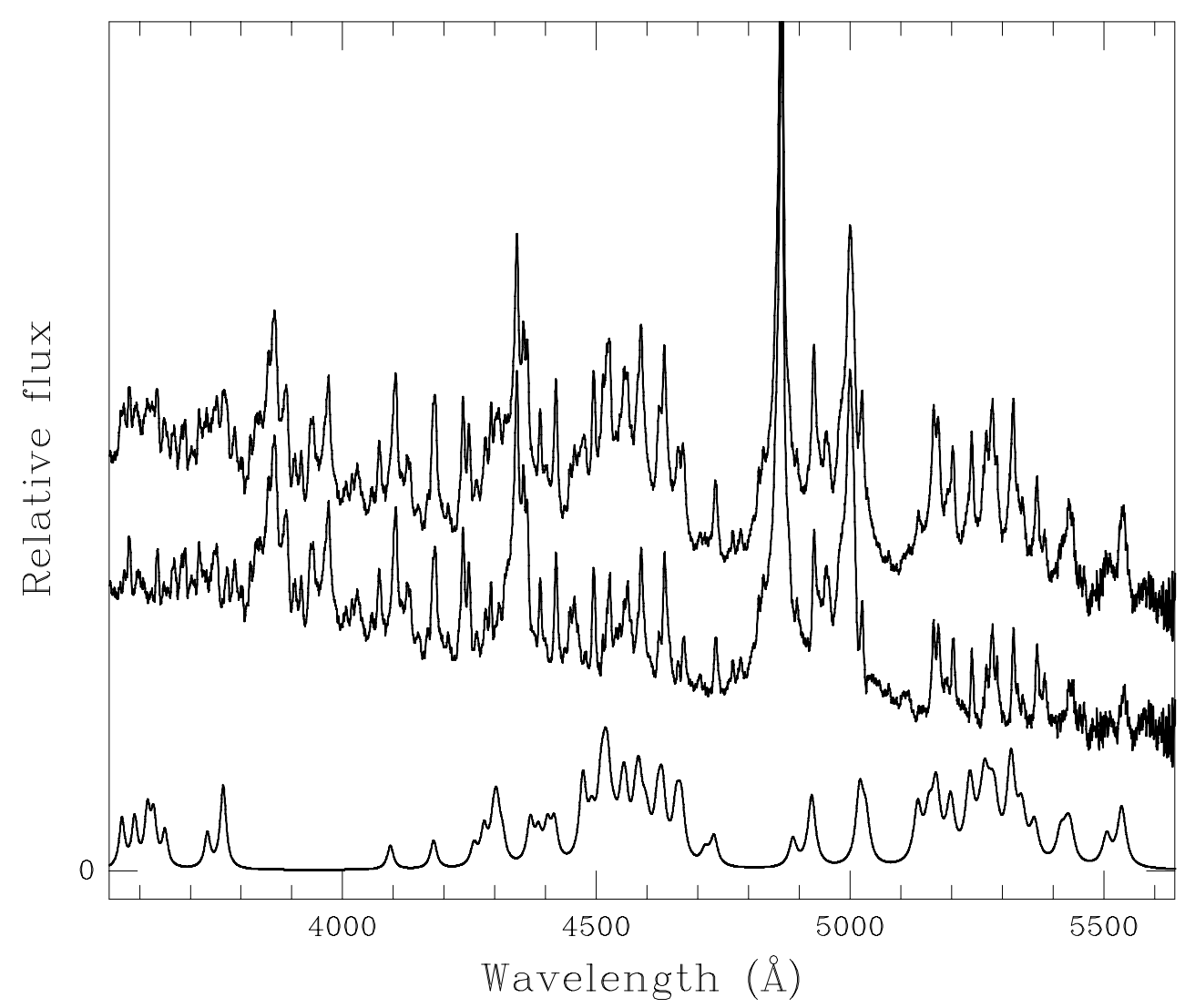

Figure 1. This figure displays the blue redshifted corrected WHT spectrum of IZw 1 (top), the Fe II template built using all Fe II lines identified in the BLR emission (bottom) and the difference (middle, shifted downward for clarity) which shows the narrow permitted and forbidden FeII lines.

$\mathrm{V}=-150 \mathrm{~km} \mathrm{~s}^{-1}$.

- A high-excitation region, moderately blueshifted: $\mathrm{V}=-500 \mathrm{~km} \mathrm{~s}^{-1}$, emitting $\mathrm{HI}$, [N II], [O III], [Ne III] with FWHM $=920 \mathrm{~km} \mathrm{~s}^{-1}$.

- Another high-excitation region with a larger blueshift: $\mathrm{V}=-1450 \mathrm{~km} \mathrm{~s}^{-1}$, emitting H I, [S II], [N II], [O III], [NeIII], [Fe VII] with FWHM $=1900 \mathrm{~km} \mathrm{~s}^{-1}$.

- And finally, a low-excitation Narrow Line Region (FWHM $=280 \mathrm{~km} \mathrm{~s}^{-1}$ ) at the systemic velocity (determined from HI measurements). It emits a number of lines from neutral elements and once ionized species including H I, Ni II, Ti II, Si II, Cr II, N I, N II, $[\mathrm{NII}],[\mathrm{S} \mathrm{II}],[\mathrm{Ca} \mathrm{II}],[\mathrm{OI}]$ plus permitted and forbidden Fe II lines: 12 [Fe II] and 75 Fe II multiplets are observed (in which 27 lines are of high excitation with $E_{u} \geqslant 10 \mathrm{eV}$ ). Altogether, 281 emission lines have been identified in the NLR. Another characteristic of this region is the absence of [O II] and [O III] lines. 


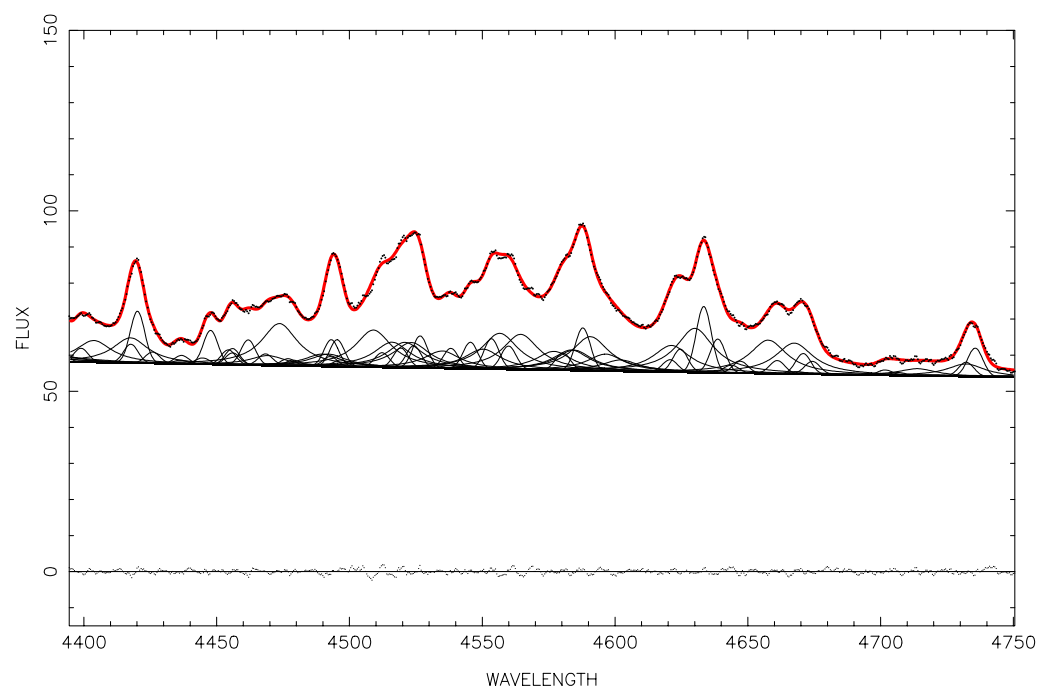

Figure 2. This figure displays the Fe II $\lambda 4570$ blend: the observed WHT spectrum superimposed to the fit (top), the residuals (bottom) and all identified individual lines included in the fit arising from the BLR and the NLR (middle).

\section{Physical Conditions in the NLR}

Based on the line ratios of the forbidden lines observed in the NLR we can infer some characteristics of this emitting region:

- $[N I I] \frac{5755}{6548+6583}$ indicates that the electron density, $\mathrm{N}_{e}$, should be larger than $10^{5}$ $\mathrm{cm}^{-3}$ (if $\left.\mathrm{T} \sim 8000 \mathrm{~K}\right)$.

- $[S I I] \frac{4069+4076}{6716+6731}$ suggests $\mathrm{N}_{e} \sim 10^{5} \mathrm{~cm}^{-3}$.

- $[O I] \frac{5577}{6300+6363}$ rather implies $\mathrm{N}_{e} \sim 10^{7} \mathrm{~cm}^{-3}$ (again if $\mathrm{T} \sim 8000 \mathrm{~K}$ ).

- Some forbidden lines such as [O I] $]$ 6300, [N II] $]$ 5755, [S II $] \lambda 4072$, [Ca II $] \lambda 7300$, [Ni II] $\lambda 7400$ have high critical densities, which suggests that electron density should be at least of the order of $10^{6}-10^{7} \mathrm{~cm}^{-3}$.

- The presence of permitted Fe II lines implies $\mathrm{N}_{e}>10^{6} \mathrm{~cm}^{-3}$, while the presence of forbidden [Fe II] lines limits this density to $10^{8} \mathrm{~cm}^{-3}$.

- Finally, the absence of $[\mathrm{O} \mathrm{II}]$ and $[\mathrm{O} \mathrm{III}]$ lines imposes a very low ionization parameter.

With such a range of parameters in mind, photoionization models have been computed using CLOUDY (Ferland 2002). The density range covers the domain $10^{5}-10^{8} \mathrm{~cm}^{-3}$ with ionization parameters in the range $10^{-7}-10^{-3}$ and a small overabundance of iron, by a factor three.

The best fits to the line intensities are obtained for models with: $10^{6}<\mathrm{N}_{e}<10^{7} \mathrm{~cm}^{-3}$ and $10^{-6}<\mathrm{U}<10^{-5}$. The distance to the central source is $\mathrm{D} \sim 1 \mathrm{kpc}$, the column density $\mathcal{N}_{H} \sim 10^{19} \mathrm{~cm}^{-2}$ and the temperature of the emission region $\mathrm{T}_{e} \sim 7000 \mathrm{~K}$. 


\section{Conclusion}

In the prototypical NLS1 galaxy I Zw 1, Fe II permitted lines are emitted by both the BLR and the NLR. The NLR is very atypical.

The Fe II template proposed here is different from that of Boroson and Green (1992) as our template includes only Fe II lines from the BLR, while the Boroson and Green (1992) one also includes the Fe II lines from the NLR together with lines from the BLR now known to be due to ions other than FeII.

More details about this work can be found in Véron-Cetty et al. (2004).

\section{References}

Boroson, T. A., \& Green, R. F. 1992, ApJS, 80, 109

Ferland, G. J, 2002 Hazy, a Brief Introduction to Cloudy, University of Kentucky, Department of Physics and Astronomy, Internal Report

Véron, P., Lindblad, P. O., Zuiderwijk, E. J., Véron-Cetty, M.-P., \& Adams, G. 1980, A\&A, 87, 245

Véron-Cetty, M.-P, Joly, M., \& Véron, P. 2004, A\&A, 417, 515 